\title{
Schwannoma in Ascending Colon: A Rare Cause of Abdominal Pain
}

\author{
Ozdal Ersoy1, Umit Sekmen², Suha Goksel3, Hulya Hamzaoglu4, Melih Paksoy² \\ ${ }^{1}$ Department of Gastroenterology, Acibadem University, Acibadem Fulya Hospital, Istanbul, Turkey \\ ${ }^{2}$ Department of Surgery, Acibadem Fulya Hospital, Istanbul, Turkey \\ ${ }^{3}$ Department of Pathology, Acibadem Maslak Hospital, Istanbul, Turkey \\ ${ }^{4}$ Department of Gastroenterology, Acibadem Fulya Hospital, Istanbul, Turkey \\ Email: ozdal.ersoy@acibadem.edu.tr
}

Received 3 March 2014; revised 2 April 2014; accepted 1 May 2014

Copyright (C) 2014 by authors and Scientific Research Publishing Inc.

This work is licensed under the Creative Commons Attribution International License (CC BY).

http://creativecommons.org/licenses/by/4.0/

c) (i) Open Access

\begin{abstract}
Schwannoma, (also called neurilemoma, neurinoma) is a benign tumor originating from nerve sheath of the Schwann cells. They rarely occur in gastrointestinal tract. We report a rare case of colonic schwannoma in a 38-year-old woman presenting with worsening right-sided pelvic pain, discovered after laparoscopic right-colon resection performed after the detection of a atypical polypoid mass in the ascending colon. Diagnosis is confirmed by immunohistochemical staining panel.
\end{abstract}

\section{Keywords}

Schwannoma, Colon, S-100

\section{Introduction}

Schwannomas, although commonly occur in the peripheral nerve of the limbs or body, spinal cord, and the central nervous system, are uncommon in gastrointestinal tract and they are benign nerve sheath tumors that arise from the Schwann cells of the neural plexus of the digestive wall. They mostly occur in stomach and in the small bowel. Isolated colonic scwannomas are rare in the literature. Few ascending colon scwannomas have been reported. Their clinical course is mostly slow and benign. They may present with hemorrhage or mechanical complications due to tumoral volume but they may rarely cause abdominal pain, too. Although rare, they must be differentiated from other intestinal mesenchymal malignancies like neurofibromas and gastrointestinal stromal tumors. Immunohistochemical staining panels are very distinctive for schwannomas, especially showing strong and diffuse positivity for S-100. The best therapeutic option is complete surgical excision with wide margins because they present the possibility of malign transformation or recur locally. The following case presentation and 
discussion highlight this rare colonic tumor and review the latest literature.

\section{Case Report}

A 38-year-old woman is referred to gastroenterology clinic from gynecology department for further evaluation of her right-sided pelvic pain. She noticed a progressive right-sided pelvic pain unresponsive to pain-killers, 3 weeks before admission. There was no change in bowel habits nor any other alarm gastrointestinal symptoms (e.g. perrectal bleeding, anemia, weight loss, loss of appetite). Her own and family medical histories are nonsignificant. She does not smoke nor use alcohol. She was taking pain-killers and spasmolytics for the last 2 weeks. On physical examination, her abdomen was soft but right quadrants of the abdomen were tender upon deep palpation. No mass was palpable. She was consultated with surgery department and acute abdominal pathology is excluded by laboratory tests and abdominal ultrasonography. Colonoscopy showed a solitary and round, wellcircumscibed, elevated polypoid lesion of $2 \mathrm{~cm}$ in diameter with an oval shaped tip and intact overlying mucosa, in the ascending colon approximately $10 \mathrm{~cm}$ away from the caecum (Figure 1). Subsequent biopsies from the tip of the lesion were composed of benign spindle cells with neuronal differentiation and strong and diffuse S-100 positivity but CD34, CD117, DOG1, SMA and desmin were all negative. The histopathology result was compatible with mucosal neuroma. Abdominopelvic tomography indicated only slight wall thickening in some parts of the caecum and ascending colon. No mass was observed. The lesion $(1.5 \times 1 \times 1 \mathrm{~cm})$ (Figure 2) was excised via laparoscopic right hemicolectomy and side-to-side ileocolonic anastomosis. Histopathological examination and immunohistochemical evaluation confirmed the lesion as schwannoma (Mucosa and submucosa is invaded. Proximal and distal surgical borders are free of tumor and reactive hyperplasia is seen in 3 excised lymphadenpathy noticed on the colon. Immunohistochemically the mass showed diffuse strong positivity for S100 (Figure 3). CD34 is also found to be positive. After the operation, she recovered well without any complication and her pelvic pain has resolved within few days. She is advised to be on follow-up for rare but possible recurrence.

\section{Discussion}

Gastrointestinal schwannomas are relatively uncommon benign tumors arising from the Schwann cells of the nerve sheath [1] [2]. They commonly originate in the stomach [3]-[5] and rarely present in large bowel. In large bowel, the commonest location being the caecum and rectosigmoid colon [6] but rare in ascending colon [7]-[9].

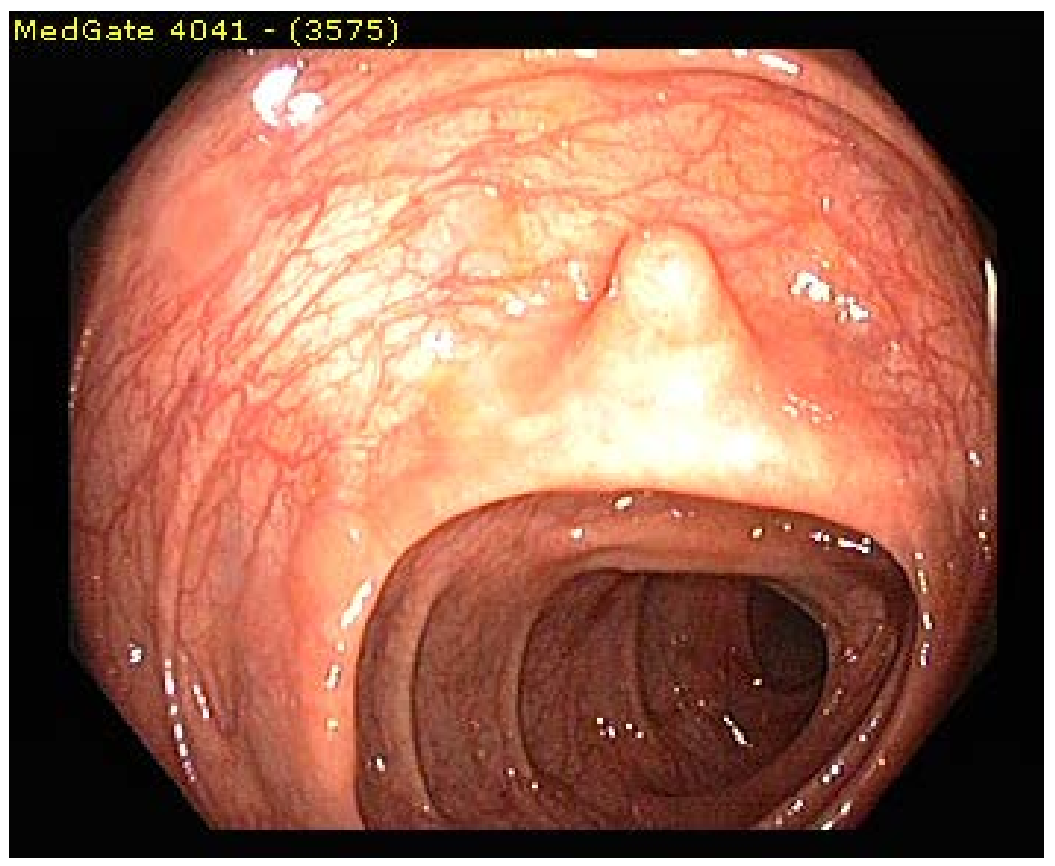

Figure 1. Colonoscopic appearance: small (approximately $1 \mathrm{~cm}$ in diameter) polypoid mass in ascending colon. 


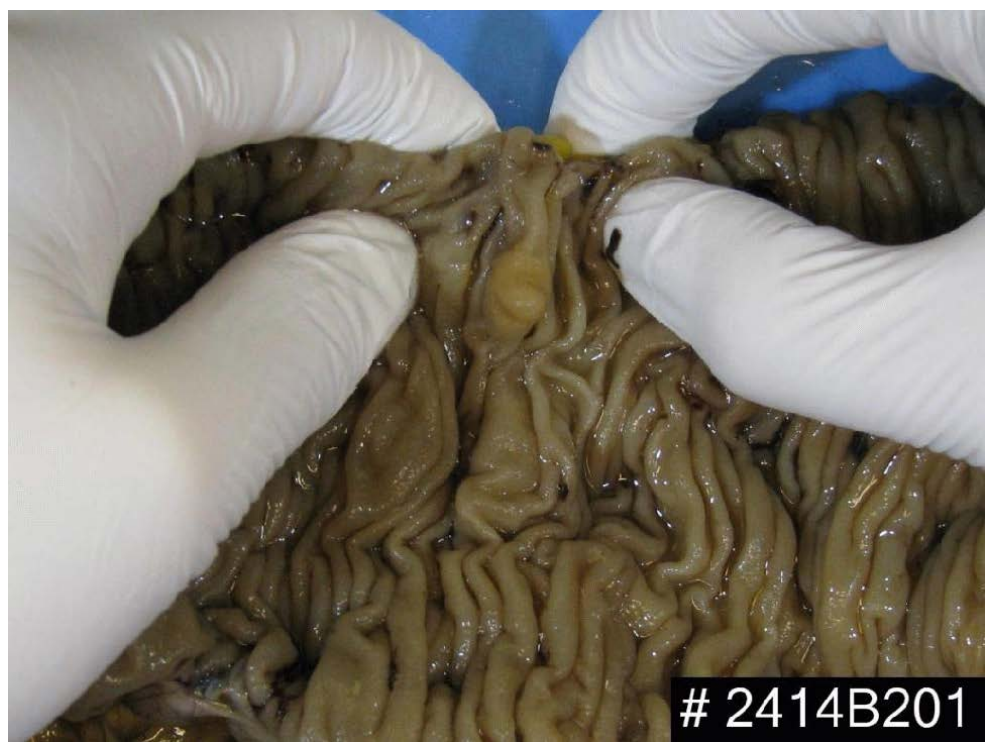

Figure 2. Macroscopic appearance $1 \times 5 \times 1 \times 1 \mathrm{~cm}$ polypoid mass.
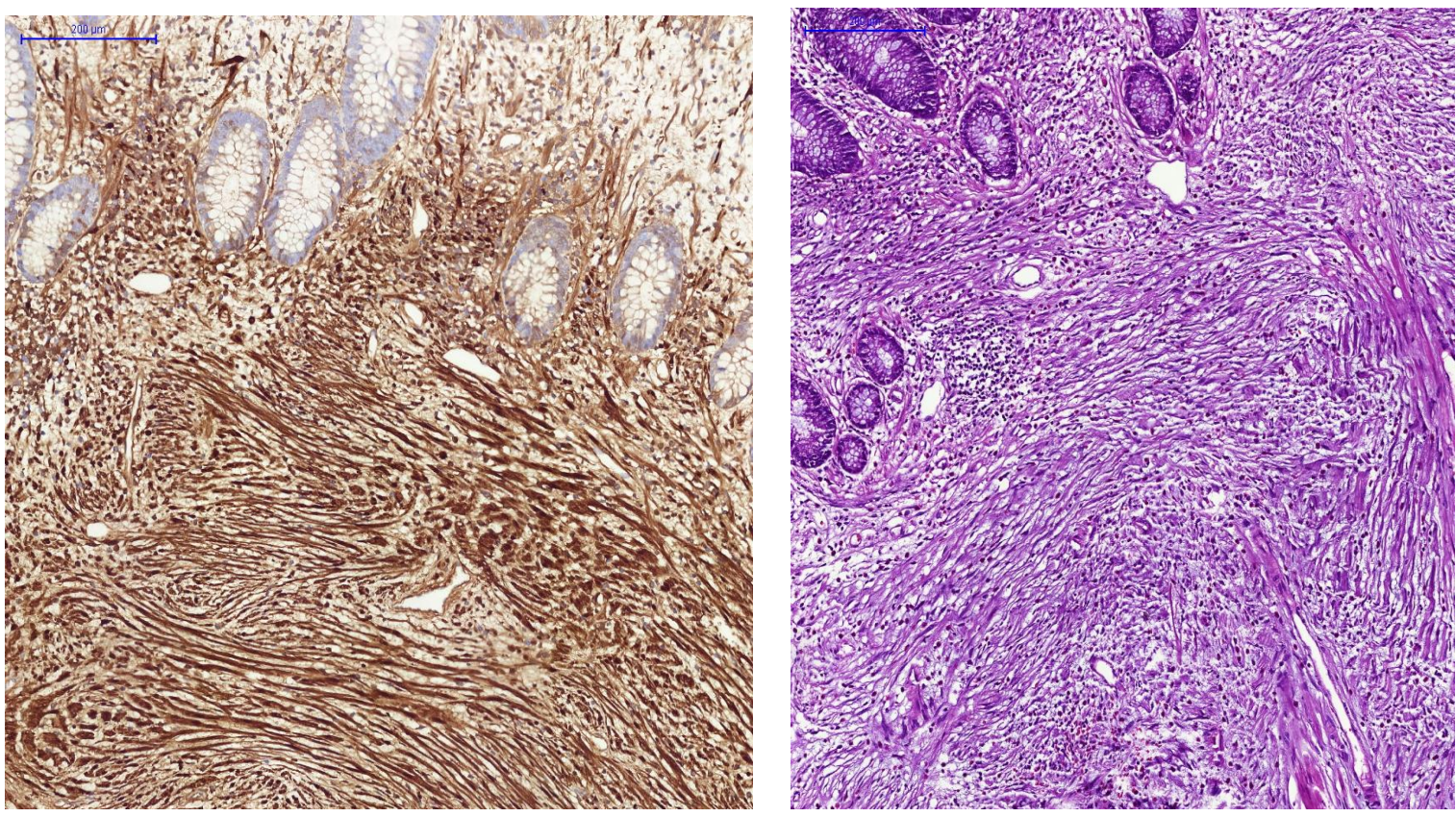

Figure 3. H\&E staining (right) S100 IHC staining (left) of the lesion $(\times 10)$.

To our knowledge, in Turkish literature, colonic schwannoma is very rare [10]. Here we report the case of a patient with schwannoma in ascending colon.

Gastrointestinal schwannoma occur equally in men and women in a wide range of age (18 - 87 years) with a mean age in the sixth to seventh decade of life [11]. However very recently Zheng et al. confirmed that gastric schwannomas have a female predominance [12]. The diagnosis is mostly established by different clinical symptoms and confirmed by radiology, endoscopic biopsies, intraoperative exploration and microscopic pathological examinations with immunohistochemical staining panel. Preoperative endoscopic biopsies and immunohistochemical studies in combination, are reliable methods for accurate preoperative diagnosis of the gastrointestinal schwannomas. After complete resection of the tumor, histologic examination is mandatory and confirmatory for the preoperative diagnosis.

According to the size and gastrointestinal location, schwannomas can cause dyspepsia, abdominal pain (co- 
licky or persistent) or discomfort, perrectal haemorrhage, anal pain, constipation, obstruction, rarely intususception [13] or a palpable mass. Some lesions can be incidentally discovered as a solitary lesion or occur synchronously with other GI malignancies [7] [8] [14] [15]. Our patient underwent colonoscopy due to new onset abdominal pain, which revealed a solitary small, polypoid mass compact with round shaped tip with $1.5-2 \mathrm{~cm}$ in diameter, located in the ascending colon. Gross features did not enable the distiction of this subepithelial lesion. Histopathological evaluation of the endoscopically taken biopsy proved the lesion to be a tumor of spindle cell proliferation obliterating mucosa and superficial submucosa, with a benign character and S-100 [SCYTEK (4c4.9)] positivity and total excision of the lesion was recommended for the accurate diagnosis.

Daimaru et al. estimated the incidence of myenteric schwannomas as being $2 \%-6 \%$ of stromal tumors of the gut in 1988 [16]. However in 2012, Diaconescu M.R and Diaconescu S. described the schwannomas as mesenchymal "non-stromal" gastrointestinal tumors in their clinical series [3]. The origin of gastrointestinal schwannomas is most likely to be from the myenteric plexus [17]. According to the World Health Organisation Classification of Tumours, gastrointestinal schwannomas are classified as nonepithelial tumors of the stomach and colon/rectum, yet are considered to be distinct, homogenous entity separate from leiomyoma, leiomyosarcoma, gastrointestinal autonomic neurogenic tumours (GANT) and gastrointestinal stromal tumours (GIST) [18]. Some authors classify gastrointestinal schwannomas as a category of GANT which are also believed to arise from Schwann cells, too [6]. In our case, the CD117 [CELLMARQUE(Y145)] negativity in the biopsy specimen ruled out the diagnosis of GIST. Preoperative endoscopic biopsies were also negative for smooth muscle actin [SCYTEK $\left(\mathrm{sm}^{-1}\right)$ ], DESMIN [SCYTEK(DE-R-1)] and CD34 [SCYTEK(Qbend/10)] performed inorder to make its differentiation from the leiomyomas and neurofibromas. Neurofibromas and leiomyomas are also composed of spindle cells however, neurofibromas show less S-100 positivity (30\% - 40\%) and leiomyomas similarly are devoid of S-100. In our case, no pleomorphism nor any ganglion cell were detected. The most probable preoperative histopathologic diagnosis of the lesion was mucosal neuroma.

Gastrointestinal schwannomas are described as homogeneously attenuated and well-defined mural masses on computerised tomography (CT) [19]. Preoperative abdominal CT of our case was not able to show the mass, and this can be explained by the small dimensions of the lesion $(1.5 \times 1 \times 1 \mathrm{~cm}$ polypoid mass). Schwannomas usually appear as solitary lesions with variable volume of 1 - 2 to 3000 grams. EUS could not be performed because of the proximal location of the polyp in the ascending colon.

Finally laparoscopic right-hemicolectomy operation was performed to excise the lesion and pathology confirmed the diagnosis of schwannoma. Immunohistochemical staining pattern of the whole tumor showed diffuse and strong positivity for S-100 [SCYTEK (4C4.9) and positivity for CD34 [SCYTEK (QBEnd/10)] Mitoses are rare in schwannomas $(<5 / 50 \mathrm{HPFs})$ and negative Kİ-67 antibodies showed no mitotic activity. Interestingly, biopsy of the lesion was negative for CD34 preoperatively therefore our case may represent a tumor with hybrid features. As most pathologists know, tumors do not read textbooks, and as a result of the differential diagnosis between schwannomas and neurofibromas can be a challenge sometime. Some authors describe a minor population of CD34-positive cells that may occur in the noncellular areas (Antoni B areas) of the schwannomas [20] which may reflect the fact that schwannoma is considered to be a tumor composed of schwann cells and little else [21]. In the series of Zheng et al., only 4 of 29 cases of gastric schwannomas showed CD34 positiviy [12].

The clinical course of the schwannomas is slow and mostly benign, but they can recur if excised incompletely and rare occasions they may present the possibility of malignant degenaration when not removed. Rare cases of malignant gastric schwannomas have been reported in the literature. Also sometimes they can mimic a malignant process, too [2]. Therefore complete resection and accurate identification of the tumor is of paramount importance and extensive surgery is not recommended [9]. In our case, tumor is resected with negative operative margins via laparoscopic right hemicolectomy.

\section{Conclusion}

As a conclusion, colonic schwannoma is a rare malignancy with a benign character and a good prognosis. Immunohistochemical staining panels are very important for definitive diagnosis and accurate preoperative diagnosis can play a great role for the most appropriate surgical approach.

\section{References}

[1] Inagawa, S., Hori, M., Shimazaki, J., Matsumoto, S., Ishii, H., Iitabashi, M., Adachi, S., Kawamoto, T. and Fukao, K. 
(2001) Solitary Schwannoma of the Colon: Report of Two Cases. Surgery Today, 31, 833-838. http://dx.doi.org/10.1007/s005950170060

[2] Skopelitou, A., Mylonakis, E., Charchanti, A. and Kappas, A. (1998) Cellular Neurilemoma (Schwannoma) of the Descending Colon Mimicking Carcinoma. Diseases of Th Colon \& Rectum, 41, 1193-1196. http://dx.doi.org/10.1007/BF02239444

[3] Diaconescu, M.R. and Diaconescu, S. (2012) Mezenchymal (Non-Epithelial) “Non-GIST” Tumors of the Digestive Tract. Chirurgia, 107, 742-750.

[4] Prevot, S., Bienvenu, L., Vaillant, J.C. and de Saint-Maur, P.P. (1999) Benign Schwannoma of the Digestive Tract: A Clinicopathologic and Immunohistochemical Study of Five Cases, Including a Case of Esophageal Tumor. The American Journal of Surgical Pathology, 23, 431-436. http://dx.doi.org/10.1097/00000478-199904000-00007

[5] Goh, B.K., Chow, P.K., Kesavan, S., Yap, W.M., Ong, H.S., Song, I.C., et al. (2008) Intraabdominal Schwannomas: A Single Institution Experience. Journal of Gastrointestinal Surgery, 12, 756-760. http://dx.doi.org/10.1007/s11605-007-0441-3

[6] Mulchandani, M.H., Chattopadhyay, D., Obafunwa, J.O. and Joypaul, V.B. (2005) Gastrointestinal Autonomic Nerve Tumors-Report of a Case and Review of the Literature. World Journal of Surgical Oncology, 3, 46. http://dx.doi.org/10.1186/1477-7819-3-46

[7] Baek, S.J., Hwangbo, W., Kim, J. and Kim, I.S. (2013) A Case of Benign Schwannoma of the Ascending Colon Treated with Laparoscopic-Assisted Wedge Resection. International Surgery, 98, 315-318. http://dx.doi.org/10.9738/INTSURG-D-13-00015.1

[8] Vasilakaki, T., Skafida, E., Arkoumani, E., Grammatoglou, X., Tsavari, K.K., Myoteri, D., Mavromati, E., Manoloudaki, K. and Zisis, D. (2012) Synchronous Primary Adenocarcinoma and Ancient Schwannoma in the Colon: An Unusual Case Report. Case Reports in Oncology, 5, 164-168.

[9] Kim, H.J., Kim, C.H., Lim, S.W., Huh, J.W., Kim, Y.J. and Kim, H.R. (2012) Schwannoma of Ascending Colon Treated by Laporoscopic Right Hemicolectomy. World Journal of Surgical Oncology, 15, 81. http://dx.doi.org/10.1186/1477-7819-10-81

[10] Calıskan, C., Akgün, E., Doganavsargil, B. and Korkut, M.A. (2009) Rare Tumors Mimicking Colorectal Carcinomas. Ege Journal of Medicine, 48, 101-107.

[11] Mysorekar, V.V., Rao, S.G., Jalihal, U. and Sridhar, M. (2010) Schwannoma of the Ascending Colon. Indian Journal of Pathology and Microbiology, 53, 198-200. http://dx.doi.org/10.4103/0377-4929.59241

[12] Zheng, L., Wu, X., Kreis, M.E., Yu, Z., Feng, L., Chen, C., Xu, B., Bu, Z., Li, Z. and Ji, J. (2014) Clinicopathological and Immunohistochemical Characterisation of Gastric Schwannomas in 29 Cases. Gastroenterology Research and Practice, 2014, Article ID: 202960.

[13] Petrie, B.A., Ho, J.M. and Tolan, A.M. (2013) Schwannoma of the Sigmoid Colon: A Rare Cause of Sigmoidorectal Intussusception. ANZ Journal of Surgery (Epub Ahead of Print). http://dx.doi.org/10.1111/ans.12364

[14] Di Cataldo, A., Trombatore, C., Cocuzza, A., Latino, R., Li Destri, G. and Petrillo, G. (2013) Synchronous Occurrence of Colon Adenocarcinoma and Gastric Schwannoma Case Report and Review of the Literature. Annali Italiani di Chirurgia, 84 (Epub Ahead of Print).

[15] Zippi, M., Pica, R., Cassieri, C., Avallone, E.V. and Occhigrossi, G. (2013) Schwannoma of the Rectum: A Case Report and Literature Review. World Journal of Clinical Cases, 16, 49-51. http://dx.doi.org/10.12998/wjcc.v1.i1.49

[16] Daimaru, Y., Kido, H., Hashimoto, H. and Enjoji, M. (1988) Benign Schwannoma of the Gastrointestinal Tract: A Clinicopathologic Immunohistochemical Study. Human Pathology, 19, 257-264. http://dx.doi.org/10.1016/S0046-8177(88)80518-5

[17] Mulchandani, M.H., Chattopadhyay, D., Obafunva, J.O. and Joypaul, V.B. (2005) Gastrointesstinal Autonomic Nerve Tumors-A Report of a Case and Review of the Literature. World Journal of Surgical Oncology, 3, 46. http://dx.doi.org/10.1186/1477-7819-3-46

[18] Wilde, B.K., Senger, J.L. and Kanthan, R. (2010) Gastrointestinal Schwannoma: An Unusual Colonic Lesion Mimicking Adenocarcinoma. Canadian Journal of Gastroenterology, 24, 233-236.

[19] Levy, A.D., Quiles, A.M., Miettinen, M. and Sobin, L.H. (2005) Gastrointestinal Schwannomas: CT Features with Clinicopathologic Correlation. American Journal of Roentgenology, 184, 797-802. http://dx.doi.org/10.2214/ajr.184.3.01840797

[20] Fletcher, C.D., Berman, J.J., Corless, C., Gorstein, F., Lasota, J., Longley, B.J., et al. (2002) Diagnosis of Gastrointestinal Tumors: A Consensus Approach. Human Pathology, 33, 459-465. http://dx.doi.org/10.1053/hupa.2002.123545

[21] Miller, R.T. (2013) Immunohistochemistry in the Differential Diagnosis of Schwannoma and Neurofibroma. www.propathlab.com 